\title{
A Note on Decomposing a Square Matrix as Sum of Two Square Nilpotent Matrices over an Arbitrary Field
}

\author{
Xiaofei Song, ${ }^{1}$ Baodong Zheng, ${ }^{1}$ and Chongguang $\mathrm{Cao}^{2}$ \\ ${ }^{1}$ Department of Mathematics, Harbin Institute of Technology, Harbin 150001, China \\ ${ }^{2}$ School of Mathematical Sciences, Heilongjiang University, Harbin 150080, China \\ Correspondence should be addressed to Baodong Zheng; zbd@hit.edu.cn
}

Received 31 August 2013; Accepted 1 October 2013

Academic Editors: A. Badawi and P. Bracken

Copyright (C) 2013 Xiaofei Song et al. This is an open access article distributed under the Creative Commons Attribution License, which permits unrestricted use, distribution, and reproduction in any medium, provided the original work is properly cited.

Let $K$ be an arbitrary field and $X$ a square matrix over $K$. Then $X$ is sum of two square nilpotent matrices over $K$ if and only if, for every algebraic extension $L$ of $K$ and arbitrary nonzero $\alpha \in L$, there exist idempotent matrices $P_{1}$ and $P_{2}$ over $L$ such that $X=\alpha P_{1}-\alpha P_{2}$.

\section{Introduction}

Botha (see [1]) proved that a square matrix $A$ over a field $K$ is a sum of two nilpotent matrices over $K$ if and only if $A$ is similar to a particular form. In an early paper, Pazzis (see [2]) gave necessary and sufficient conditions in which a matrix can be decomposed as a linear combination of two idempotents with given nonzero coefficients. The goal of this paper is to build a bridge that connects the result obtained in [1] with the result obtained in [2]. However, the relation between these two facts has not been formally discussed yet (more details in [3-9]).

If there is no statement, the meanings of notations mentioned in this paragraph hold all over the paper. $K$ denotes an arbitrary field, $\bar{K}$ is its algebraic closure, $L$ is an arbitrary algebraic extension of $K$, and $\operatorname{car}(K)$ is the characteristic of $K$. $Z^{+}$denotes the set of all positive integers, $[s]=\left\{z \in Z^{+} \mid 1 \leq\right.$ $z \leq s\}$ for some $s \in Z^{+} . M_{m, n}(K)$ denotes the space consisting of all $m \times n$ matrices over $K ; M_{n}(K)=M_{n, n}(K) . r(A)$ is the rank of $A \in M_{m, n}(K)$. $E$ denotes a vector space over $K$ and $\operatorname{dim}(E)$ is the dimension of $E . X \in M_{n}(K)$ is called ${ }_{s}^{2} N$ in $M_{n}(K)$ if there exist square nilpotent $N_{1}$ and $N_{2} \in M_{n}(K)$ such that $X=N_{1}+N_{2}$, while $X$ is called an $(\alpha, \beta)$ composite in $M_{n}(K)$ if there exist idempotent $P_{1}$ and $P_{2} \in M_{n}(K)$ such that $X=\alpha P_{1}+\beta P_{2}$, where $\alpha, \beta \in K \backslash\{0\}$ (Definition 1 in [2]); in particular, $X$ is called ${ }_{ \pm} P$ if $X$ is an $(\alpha,-\alpha)$ composite in $M_{n}(L)$ for every algebraic extension $L$ of $K$ and arbitrary nonzero $\alpha \in L$ (when $\operatorname{car}(K)=2$, we still use ${ }_{ \pm} P$ for the meaning of $(\alpha, \alpha)$ composites).
For $X \in M_{n}(K)$, on the one hand, we will prove that $X$ is ${ }_{s}^{2} N$ in $M_{n}(K)$ implies $X$ is ${ }_{ \pm} P$; that is, the form provided by Botha satisfies the condition as in [2]; on the other hand, we will also prove that $X$ is ${ }_{+} P$ implies $X$ is ${ }_{s}^{2} N$ in $M_{n}(K)$; that is, we can derive the form provided in [1] from the results obtained in [2]. In fact, the following theorem is the main result of this paper.

Theorem 1 (main theorem). Suppose $K$ is an arbitrary field and $X \in M_{n}(K)$; then $X$ is ${ }_{s}^{2} N$ in $M_{n}(K)$ if and only if $X$ is ${ }_{ \pm} P$.

In Section 2, we will state some related theorems and notations from [2] and we will give some necessary corollaries. The proof of Theorem 1 will be carried out in Section 3.

\section{More Notations and Necessary Corollaries}

Suppose $X \in M_{n}(K)$ and $X_{i} \in M_{n_{i}}(K)$, we denote by $X=$ $X_{1} \oplus \cdots \oplus X_{s}$ the following matrix with $\sum_{i=1}^{s} n_{i}=n$ :

$$
\left(\begin{array}{llll}
X_{1} & & & \\
& X_{2} & & \\
& & \ddots & \\
& & & X_{s}
\end{array}\right) .
$$

Notation 1 (Notation 2 in [2]). Let $X \in M_{n}(K), \lambda \in \bar{K}$ and $k \in Z^{+}$; we denote by 
(1) $j_{k}(X, \lambda)$ the number of blocks of size $k$ for the eigenvalue $\lambda$ in the Jordan reduction of $X$;

(2) $n_{k}(X, \lambda)$ the number of blocks of size greater or equal to $k$ for the eigenvalue $\lambda$ in the Jordan reduction of $X$.

Definition 2 (Definition 3 in [2]). Two sequences $\left(u_{k}\right)_{k \geq 1}$ and $\left(v_{k}\right)_{k \geq 1}$ are side to be intertwined if for all $k \in Z^{+}, v_{k} \geq u_{k+1}$, and $u_{k} \geq v_{k+1}$.

Notation 2 (Notation 4 in [2]). Given a monic polynomial, $P=x^{n}-a_{n-1} x^{n-1}-\cdots-a_{1} x-a_{0}$, denote the following $C(P)$ by its companion matrix:

$$
C(P)=\left(\begin{array}{cccccc}
0 & 0 & \cdots & \cdots & 0 & a_{0} \\
1 & 0 & 0 & \cdots & 0 & a_{1} \\
0 & 1 & 0 & \ddots & 0 & a_{2} \\
\vdots & \ddots & \ddots & \ddots & \ddots & \vdots \\
\vdots & \cdots & \ddots & 1 & 0 & a_{n-2} \\
0 & \cdots & \cdots & 0 & 1 & a_{n-1}
\end{array}\right)
$$

Theorem 3 (Theorem 1 in [2]). Assume $\operatorname{car}(K) \neq 2$ and let $X \in M_{n}(K)$. Then $X$ is an $(\alpha,-\alpha)$ composite if and only if all the following conditions hold.

(1) The sequences $\left(n_{k}(X, \alpha)\right)_{k \geq 1}$ and $\left(n_{k}(X,-\alpha)\right)_{k \geq 1}$ are intertwined;

(2) for all $\lambda \in \bar{K} \backslash\{0, \alpha,-\alpha\}$ and for all $k \in Z^{+}, j_{k}(X, \lambda)=$ $j_{k}(X,-\lambda)$.

Theorem 4 (Theorem 5 in [2]). Assume car $(K)=2$ and let $X \in M_{n}(K)$. Then $X$ is an $(\alpha,-\alpha)$ composite if and only if for every $\lambda \in \bar{K} \backslash\{0, \alpha\}$, all blocks in the Jordan reduction of $X$ with respect to $\lambda$ have an even size.

Suppose $X \in M_{n}(k)$ is ${ }_{+} P$, where $\operatorname{car}(K) \neq 2$. Then $X$ is $(\alpha,-\alpha)$ composite and $(\beta,-\bar{\beta})$ composite in $M_{n}(L)$ for some algebraic extension $L$ of $K$, where $\alpha, \beta \in L \backslash\{0\}$ with $\alpha \neq \pm \beta$. By Theorem 3, the following statements are true:

(1) for all $\lambda \in \bar{K} \in\{0, \alpha,-\alpha\}$ and for all $k \in Z^{+}$, $j_{k}(X, \lambda)=j_{k}(X,-\lambda)$;

(2) for all $\lambda \in \bar{K} \in\{0, \beta,-\beta\}$ and for all $k \in Z^{+}$, $j_{k}(X, \lambda)=j_{k}(X,-\lambda)$.

so for all $\lambda \in \bar{K} \backslash\{0\}$ and for all $k \in Z^{+}, j_{k}(X, \lambda)=j_{k}(X,-\lambda)$.

On the other hand, note that for nonzero $\alpha \in \bar{K}$ with $\operatorname{car}(K) \neq 2$, the sequences $\left(n_{k}(X, \alpha)\right)_{k \geq 1}$ and $\left(n_{k}(X,-\alpha)\right)_{k \geq 1}$ are intertwined if for all $k \in Z^{+}, j_{k}(X, \alpha)=j_{k}(X,-\alpha)$. Then for all $\lambda \in \bar{K} \backslash\{0\}, k \in Z^{+}, j_{k}(X, \lambda)=j_{k}(X,-\lambda)$ implies that for every algebraic extension $L$ of $K$ and arbitrary nonzero $\alpha \in L, X$ is an $(\alpha,-\alpha)$ composite in $M_{n}(L)$; that is, $X$ is ${ }_{ \pm} P$.

Therefore the following corollary is true.

Corollary 5. Assume $\operatorname{car}(K) \neq 2$ and let $X \in M_{n}(K)$. Then $X$ is ${ }_{ \pm} P$ if and only if for all $\lambda \in \bar{K} \backslash\{0\}$ for all $k \in Z^{+}$, $j_{k}(X, \bar{\lambda})=j_{k}(X,-\lambda)$.

Similarly, we can derive the following corollary from Theorem 4.
Corollary 6. Assume $\operatorname{car}(K)=2$ and let $X \in M_{n}(K)$. Then $X$ is ${ }_{ \pm} P$ if and only if for every $\lambda \in \bar{K} \backslash\{0\}$, all blocks in the Jordan reduction of $X$ with respect to $\lambda$ have an even size.

Naturally, we derive the following corollary from the above two corollaries.

Corollary 7. Every nilpotent is ${ }_{ \pm} P$.

In fact, arbitrary nilpotent is not only ${ }_{ \pm} P$ but also ${ }_{s}^{2} N$.

Lemma 8. Every nilpotent $N \in M_{n}(K)$ is ${ }_{s}^{2} N$.

Proof. For arbitrary field $K$, let $N \in M_{n}(K)$ is nilpotent; then $N$ is similar to $N_{1} \oplus N_{2} \oplus \cdots \oplus N_{s}$, where for every $i \in[s], N_{i} \in$ $M_{r_{i}}(K), \sum_{i=1}^{s} r_{i}=n$, and both the characteristic polynomial and minimal polynomial of $N_{i}$ are $x^{r_{i}}$. Furthermore, $N_{i}$ is similar to $C\left(x^{r_{i}}\right)$ as follows:

$$
\left(\begin{array}{cccc}
0 & 0 & \cdots & 0 \\
1 & 0 & \cdots & 0 \\
\vdots & \ddots & \ddots & \vdots \\
0 & \cdots & 1 & 0
\end{array}\right)_{r_{i} \times r_{i}}
$$

That is, $C\left(x^{r_{i}}\right)=E_{2,1}+E_{3,2}+\cdots+E_{r_{i}, r_{i}-1} \in M_{r_{i}}(K)$.

When $r_{i}$ is even, $C\left(x^{r_{i}}\right)=\sum_{j=1}^{r_{i} / 2} E_{2 j, 2 j-1}+\sum_{j=1}^{r_{i} / 2-1} E_{2 j+1,2 j}$; when $r_{i}$ is odd, $C\left(x^{r_{i}}\right)=\sum_{j=1}^{\left(r_{i}-1\right) / 2} E_{2 j, 2 j-1}+\sum_{j=1}^{\left(r_{i}-1\right) / 2} E_{2 j+1,2 j}$. Note that both $\sum E_{2 j, 2 j-1}$ and $\sum E_{2 j+1,2 j}$ are square nilpotent matrices then $C\left(x^{r_{i}}\right)$ is ${ }_{s}^{2} N$, and $N_{i}$ is ${ }_{s}^{2} N$ follows. Hence $N$ is ${ }_{s}^{2} N$.

\section{Proof of Main Theorem}

${ }_{s}^{2} N \rightarrow{ }_{ \pm} P$. Suppose $X \in M_{n}(K)$ is ${ }_{s}^{2} N$ in $M_{n}(K)$; that is, there exist square nilpotent matrices $N_{1}$ and $N_{2} \in M_{n}(K)$ such that $X=N_{1}+N_{2}$. It will take two steps to prove $X$ is ${ }_{ \pm} P$.

Step 1. If $X$ is nonsingular, then $X$ is ${ }_{ \pm} P$.

Since $X=N_{1}+N_{2}$ with $N_{1}^{2}=N_{2}^{2}=0$, inspect the eigenspaces of $N_{1}$ and $N_{2}$. Note that $N_{1}$ and $N_{2}$ are square nilpotent matrices, their ranks satisfy the following inequality matrices.

$$
r\left(N_{1}\right)+r\left(N_{2}\right) \leq n,
$$

where equality holds if and only if $r\left(N_{1}\right)=r\left(N_{2}\right)=n / 2$.

At first, $X$ is nonsingular implies 0 is not its eigenvalue. Secondly, if the inequality is strict, then intersection of eigenspaces of $N_{1}$ and $N_{2}$ contains nonzero vectors; that is, there exists nonzero $x \in M_{n, 1}(K)$ such that $N_{1} x=N_{2} x=0$, which implies that 0 is one of eigenvalues of $X$. This is a contradiction. Hence, $r\left(N_{1}\right)+r\left(N_{2}\right)=n$; that is, $n$ is even and $N_{1}$ and $N_{2}$ are similar but not equal.

Because $N_{1}$ is square nilpotent with $r\left(N_{1}\right)=n / 2$, we can choose $n / 2$ linear independent vectors from the set of its column vectors which can make up a base of eigenspace of $N_{1}$ and denote $\beta$ by the $n \times(n / 2)$ matrix consisting of these $n / 2$ columns. Correspondingly, we have $n \times(n / 2)$ matrix $\gamma$ with 
all columns from the set of columns of $N_{2}$. Because 0 is the only vector in the intersection of eigenspaces of $N_{1}$ and $N_{2}$, $n \times n$ matrix $(\beta \gamma)$ is nonsingular.

$N_{1}^{2} \gamma=0$ implies that nonzero column vectors of $N_{1} \gamma$ are eigenvectors of $N_{1}$ and $N_{1}\left(\begin{array}{ll}\beta & \gamma\end{array}\right)=\left(\begin{array}{ll}0 & N_{1} \gamma\end{array}\right)$ implies $r\left(N_{1} \gamma\right)=n / 2$. Hence; $N_{1} \gamma$ and $\beta$ are equal under certain column transformation; that is, there is an invertible matrix $T_{1}$ such that $N_{1} \gamma=\beta T_{1}$. Correspondingly, there is an invertible matrix $T_{2}$ such that $N_{2} \beta=\gamma T_{2}$.

Let $\left(\begin{array}{l}y_{1} \\ y_{2}\end{array}\right)$ be the inverse of $(\beta \gamma)$, where $y_{1}$ and $y_{2}$ are $(n / 2) \times n$ matrices. Naturally, the following equation is true:

$$
\left(\begin{array}{l}
y_{1} \\
y_{2}
\end{array}\right)\left(\begin{array}{ll}
\beta & \gamma
\end{array}\right)=\left(\begin{array}{ll}
y_{1} \beta & y_{1} \gamma \\
y_{2} \beta & y_{2} \gamma
\end{array}\right)=\left(\begin{array}{ll}
I_{n / 2} & 0_{n / 2} \\
0_{n / 2} & I_{n / 2}
\end{array}\right)
$$

Now, we carry out the same similarity transformation on $N_{1}$ and $N_{2}$ as follows:

$$
\begin{aligned}
& \left(\begin{array}{l}
y_{1} \\
y_{2}
\end{array}\right) N_{1}\left(\begin{array}{ll}
\beta & \gamma
\end{array}\right)=\left(\begin{array}{ll}
y_{1} N_{1} \beta & y_{1} N_{1} \gamma \\
y_{2} N_{1} \beta & y_{2} N_{1} \gamma
\end{array}\right), \\
& \left(\begin{array}{l}
y_{1} \\
y_{2}
\end{array}\right) N_{2}\left(\begin{array}{ll}
\beta & \gamma
\end{array}\right)=\left(\begin{array}{ll}
y_{1} N_{2} \beta & y_{1} N_{2} \gamma \\
y_{2} N_{2} \beta & y_{2} N_{2} \gamma
\end{array}\right) .
\end{aligned}
$$

Note that $N_{1} \gamma=\beta T_{1}$ and $N_{2} \beta=\gamma T_{2}$, the above three equations imply that $N_{1}$ is similar to $\left(\begin{array}{cc}0_{n / 2} & T_{1} \\ 0_{n / 2} & 0_{n / 2}\end{array}\right) \in M_{n}(K)$ and $N_{2}$ is similar to $\left(\begin{array}{cc}0_{n / 2} & 0_{n / 2} \\ T_{2} & 0_{n / 2}\end{array}\right) \in M_{n}(K)$.

Hence, $X$ is similar to $\left(\begin{array}{cc}0_{n / 2} & T_{1} \\ 0_{n / 2} & 0_{n / 2}\end{array}\right)+\left(\begin{array}{cc}0_{n / 2} & 0_{n / 2} \\ T_{2} & 0_{n / 2}\end{array}\right)$. For every algebraic extension $L$ of $K$ and arbitrary nonzero $\alpha \in L, X$ is also similar to the following matrix:

$$
\alpha\left(\begin{array}{cc}
I_{n / 2} & \alpha^{-1} T_{1} \\
0_{n / 2} & 0_{n / 2}
\end{array}\right)-\alpha\left(\begin{array}{cc}
I_{n / 2} & 0_{n / 2} \\
-\alpha^{-1} T_{2} & 0_{n / 2}
\end{array}\right)
$$

That is, $X$ is ${ }_{ \pm} P$.

Step 2. If $X$ is singular and similar to $Y \oplus N$, where $Y$ is nonsingular and $N$ is nilpotent. Then $X$ is ${ }_{+} P$.

At first, we need to prove that $Y$ is ${ }_{s}^{2} N$. Without loss of generality, we assume $X=Y \oplus N$ in the following proof since ${ }_{s}^{2} N$ holds under similarity transformations.

Let $N_{1}=\left(\begin{array}{ll}n_{1} & n_{2} \\ n_{3} & n_{4}\end{array}\right)$, where the order of $n_{1}$ is the same for $Y$ and the order of $n_{4}$ is the same for $N$. Then $N_{1}^{2}=0$ implies the following equations are true:

$$
\begin{array}{ll}
n_{1}^{2}+n_{2} n_{3}=0, & n_{1} n_{2}+n_{2} n_{4}=0 \\
n_{3} n_{1}+n_{4} n_{3}=0, & n_{3} n_{2}+n_{4}^{2}=0 .
\end{array}
$$

Since $\left(X-N_{1}\right)^{2}=N_{2}^{2}=0$, we get the following equations after replacing $n_{1}$ with $Y-n_{1}$ and $n_{4}$ with $N-n_{4}$ in the previous equations:

$$
\begin{gathered}
\left(Y-n_{1}\right)^{2}+n_{2} n_{3}=0, \\
\left(Y-n_{1}\right) n_{2}+n_{2}\left(N-n_{4}\right)=0, \\
n_{3}\left(Y-n_{1}\right)+\left(N-n_{4}\right) n_{3}=0, \\
n_{3} n_{2}+\left(N-n_{4}\right)^{2}=0 .
\end{gathered}
$$

We can derive the following equations from the $3 \mathrm{rd}$ and 4th equations in the above two sets of equations:

$$
Y n_{2}+n_{2} N=0, \quad n_{3} Y+N n_{3}=0
$$

Note that $N$ is nilpotent, assume its index is $r$; that is, $N^{r-1} \neq 0$ and $N^{r}=0$. After multiplying the right side of equation $Y n_{2}+n_{2} N=0$ by $N^{r-1}$, we can get $Y n_{2} N^{r-1}=0 . Y$ is nonsingular implies $n_{2} N^{r-1}=0$. Repeat the operation, we eventually get $n_{2}=0$. Similarly, we can also get $n_{3}=0$.

So $N_{1}$ is quasidiagonal and $N_{2}$ is also quasidiagonal through similar proof; that is, $n_{1}$ and $n_{4}$ are square nilpotent same as the corresponding parts of $N_{2}$. Finally, we prove that $Y$ is ${ }_{s}^{2} N$.

Since $Y$ is ${ }_{ \pm} P$ by Step 1 and $N$ is ${ }_{ \pm} P$ by Corollary 7 , it is true that $X$ is ${ }_{ \pm} P$.

${ }_{ \pm} P \rightarrow{ }_{s}^{2} N$. Suppose $X \in M_{n}(K)$ is ${ }_{ \pm} P$. If $X$ is similar to $Y \oplus \bar{N}$, where $Y$ is nonsingular and $N$ is nilpotent, then $X$ is ${ }_{ \pm} P$ if and only if $Y$ is ${ }_{+} P$ by Corollaries 5, 6, and 7. Without loss of generality, we can assume $X$ is nonsingular. Furthermore, if $X$ is nonsingular and similar to $Y_{1} \oplus Y_{2}$, where all eigenvalues of $Y_{1}$ are not in $K$ and all eigenvalues of $Y_{2}$ are in $K$. Then $X$ is ${ }_{ \pm} P$ if and only if $Y_{1}$ is ${ }_{ \pm} P$ and $Y_{2}$ is ${ }_{ \pm} P$. It will take two steps to prove $X$ is ${ }_{s}^{2} N$.

Step 3. Suppose $\operatorname{car}(K) \neq 2$ and all eigenvalues of $X$ are not in $K$; then for arbitrary nonzero $\alpha \in K, X$ is an $(\alpha,-\alpha)$ composite; that is, there exist idempotent matrices $P_{1}$ and $P_{2} \in M_{n}(K)$ such that $X=\alpha P_{1}-\alpha P_{2}$.

Let $Q_{1}^{(0)}$ be the eigenspace of $P_{1}$ with respect to $0, Q_{1}^{(1)}$ the eigenspace of $P_{1}$ with respect to 1 , let $Q_{2}^{(0)}$ be the eigenspace of $P_{2}$ with respect to 0 , and $Q_{2}^{(1)}$ the eigenspace of $P_{2}$ with respect to 1 . Both $\alpha$ and $-\alpha$ are not eigenvalues of $X$ implies that $Q_{1}^{(0)} \cap Q_{2}^{(0)}=Q_{1}^{(1)} \cap Q_{2}^{(1)}=Q_{1}^{(0)} \cap Q_{2}^{(1)}=Q_{1}^{(1)} \cap Q_{2}^{(0)}=\{0\}$; then $\operatorname{dim}\left(Q_{1}^{(0)}\right)=\operatorname{dim}\left(Q_{1}^{(1)}\right)=\operatorname{dim}\left(Q_{2}^{(0)}\right)=\operatorname{dim}\left(Q_{2}^{(1)}\right)=$ $n / 2$ (otherwise, $\operatorname{dim}\left(Q_{1}^{(0)}\right) \geq n / 2$ implies $Q_{1}^{(0)} \cap Q_{2}^{(0)} \neq\{0\}$ or $Q_{1}^{(0)} \cap Q_{2}^{(1)} \neq\{0\}$, etc.); that is, $n$ is even.

Suppose $S$ and $T$ are $n \times(n / 2)$ matrices with $r(S)=r(T)=$ $n / 2$ satisfying $P_{1} S=0$ and $P_{2} T=T$; then $(S T)$ is $n \times n$ nonsingular matrix. Let $\left(\begin{array}{l}U \\ V\end{array}\right)$ be its inverse; that is,

$$
\left(\begin{array}{l}
U \\
V
\end{array}\right)\left(\begin{array}{ll}
S & T
\end{array}\right)=\left(\begin{array}{cc}
U S & U T \\
V S & V T
\end{array}\right)=\left(\begin{array}{cc}
I_{n / 2} & 0_{n / 2} \\
0_{n / 2} & I_{n / 2}
\end{array}\right) .
$$

Then we carry out the same similarity transformation on $P_{1}$ and $P_{2}$ as follows:

$$
\begin{aligned}
& \left(\begin{array}{l}
U \\
V
\end{array}\right) P_{1}\left(\begin{array}{ll}
S & T
\end{array}\right)=\left(\begin{array}{ll}
U P_{1} S & U P_{1} T \\
V P_{1} S & V P_{1} T
\end{array}\right)=\left(\begin{array}{ll}
0_{n / 2} & U P_{1} T \\
0_{n / 2} & V P_{1} T
\end{array}\right), \\
& \left(\begin{array}{l}
U \\
V
\end{array}\right) P_{2}\left(\begin{array}{ll}
S & T
\end{array}\right)=\left(\begin{array}{ll}
U P_{2} S & U P_{2} T \\
V P_{2} S & V P_{2} T
\end{array}\right)=\left(\begin{array}{ll}
U P_{2} S & 0_{n / 2} \\
V P_{2} S & I_{n / 2}
\end{array}\right),
\end{aligned}
$$

where $P_{1}$ and $P_{2}$ are idempotent implies that $V P_{1} T$ and $U P_{2} S$ are idempotent and $r\left(P_{1}\right)=r\left(P_{2}\right)=n / 2$ implies that $V P_{1} T=$ $I_{n / 2}$ and $U P_{2} S=0_{n / 2}$. Hence, $X$ is similar to the following matrix:

$$
\left(\begin{array}{cc}
0_{n / 2} & \alpha U P_{1} T \\
0_{n / 2} & 0_{n / 2}
\end{array}\right)+\left(\begin{array}{cc}
0_{n / 2} & 0_{n / 2} \\
-\alpha V P_{2} S & 0_{n / 2}
\end{array}\right) .
$$

That is, $X$ is ${ }_{s}^{2} N$ in $M_{n}(K)$. 
When $\operatorname{car}(K)=2, X$ is $(\alpha, \alpha)$ composite for arbitrary nonzero $\alpha \in K$, we can similarly prove that $X$ is ${ }_{s}^{2} N$ in $M_{n}(K)$ replacing $-\alpha$ with $\alpha$ in the previous proof.

Step 4. Suppose $\operatorname{car}(K) \neq 2$ and all eigenvalues of $X$ are in $K$; then by Corollary $5, j_{k}(X, \alpha)=j_{k}(X,-\alpha)$ for every $k \in Z^{+}$ and arbitrary nonzero $\alpha \in K$.

Moreover, $X$ is similar to $X_{1} \oplus \cdots \oplus X_{s}$, where both the characteristic polynomial and the minimal polynomial of $X_{i}$ are $\left[\left(x-\alpha_{i}\right)\left(x+\alpha_{i}\right)\right]^{r_{i}}=\left(x^{2}-\alpha_{i}^{2}\right)^{r_{i}}$ with $2 \sum_{i=1}^{s} r_{i}=n$ and $\alpha_{i} \in K \backslash\{0\}$ is one of eigenvalues of $X$ for every $i \in[s]$. Without loss of generality, we just need to prove $X_{i}$ is ${ }_{s}^{2} N$.

Since $X_{i}$ is similar to $C\left(\left(x^{2}-\alpha_{i}^{2}\right)^{r_{i}}\right)$ as follows:

$$
\left(\begin{array}{cccccc}
0 & 0 & \cdots & \cdots & 0 & a_{0} \\
1 & 0 & \cdots & \cdots & 0 & 0 \\
0 & 1 & 0 & \cdots & 0 & a_{2} \\
\vdots & \ddots & \ddots & \ddots & \vdots & \vdots \\
\vdots & \ddots & \ddots & 1 & 0 & a_{2 r_{i}-2} \\
0 & \cdots & \cdots & 0 & 1 & 0
\end{array}\right),
$$

where $\left(x^{2}-\alpha_{i}^{2}\right)^{r_{i}}=x^{2 r_{i}}-a_{2 r_{i}-2} x^{2 r_{i}-2}-\cdots-a_{2} x^{2}-a_{0}$. We have $C\left(\left(x^{2}-\alpha_{i}^{2}\right)^{r_{i}}\right)=E_{2,1}+\cdots+E_{2 r_{i}, 2 r_{i}-1}+a_{0} E_{1,2 r_{i}}+a_{2} E_{3,2 r_{i}}+$ $\cdots+a_{2 r_{i}-2} E_{2 r_{i}-1,2 r_{i}}=\left(E_{2,1}+E_{4,3}+\cdots+E_{2 r_{i}, 2 r_{i}-1}\right)+\left(E_{3,2}+\cdots+\right.$ $\left.E_{2 r_{i}-1,2 r_{i}-2}+a_{0} E_{1,2 r_{i}}+a_{2} E_{3,2 r_{i}}+\cdots+a_{2 r_{i}-2} E_{2 r_{i}-1,2 r_{i}}\right)=N_{1}+N_{2}$. Obviously, both $N_{1}$ and $N_{2}$ are square nilpotent matrices; that is, $X_{i}$ is ${ }_{s}^{2} N$. Hence, $X$ is ${ }_{s}^{2} N$ in $M_{n}(K)$.

When $\operatorname{car}(K)=2$, all blocks in the Jordan reduction of $X$ with respect to $\alpha \in K \backslash\{0\}$ have an even size by Corollary 6; that is, both the characteristic polynomial and minimal polynomial of every block with respect to $\alpha$ are $(x+\alpha)^{s_{i}}=\left((x+\alpha)^{2}\right)^{s_{i} / 2}=\left(x^{2}+\alpha^{2}\right)^{s_{i} / 2}$, where $s_{i}$ is even. Similarly, we can also prove that $X$ is ${ }_{s}^{2} N$ in $M_{n}(K)$.

\section{References}

[1] J. D. Botha, "Sums of two square-zero matrices over an arbitrary field," Linear Algebra and Its Applications, vol. 436, no. 3, pp. 516-524, 2012.

[2] C. D. Pazzis, "On linear combinations of two idempotent matrices over an arbitrary field," Linear Algebra and Its Applications, vol. 433, no. 3, pp. 625-636, 2010.

[3] R. E. Hartwig and M. S. Putcha, "When is a matrix a difference of two idempotents?" Linear and Multilinear Algebra, vol. 26, no. 4, pp. 267-277, 1990.

[4] J. H. Wang and P. Y. Wu, "Sums of square-zero operators," Studia Mathematica, vol. 99, no. 2, pp. 115-127, 1991.

[5] F. R. Gantmacher, The Theory of Matrices, vol. 1, Chelsea, New York, NY, USA, 1959.

[6] H. Flanders, "Elementary divisors of $A B$ and $B A$," Proceedings of the American Mathematical Society, vol. 2, no. 6, pp. 871-874, 1951.

[7] W. E. Roth, "The equations $A X-Y B=C$ and $A X-X B=C$ in matrices," Proceedings of the American Mathematical Society, vol. 3, pp. 392-396, 1952.

[8] C. Pearcy and D. Topping, "Sums of small numbers of idempotents," The Michigan Mathematical Journal, vol. 14, pp. 453-465, 1967.
[9] R. E. Hartwig and M. S. Putcha, "When is a matrix a sum of idempotents?" Linear and Multilinear Algebra, vol. 26, no. 4, pp. 279-286, 1990. 


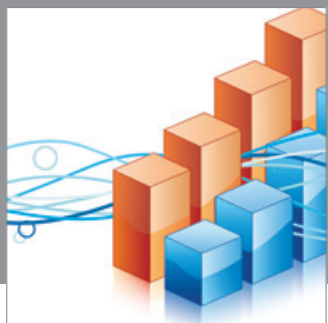

Advances in

Operations Research

mansans

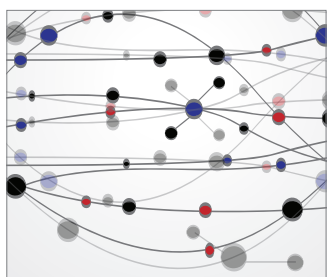

The Scientific World Journal
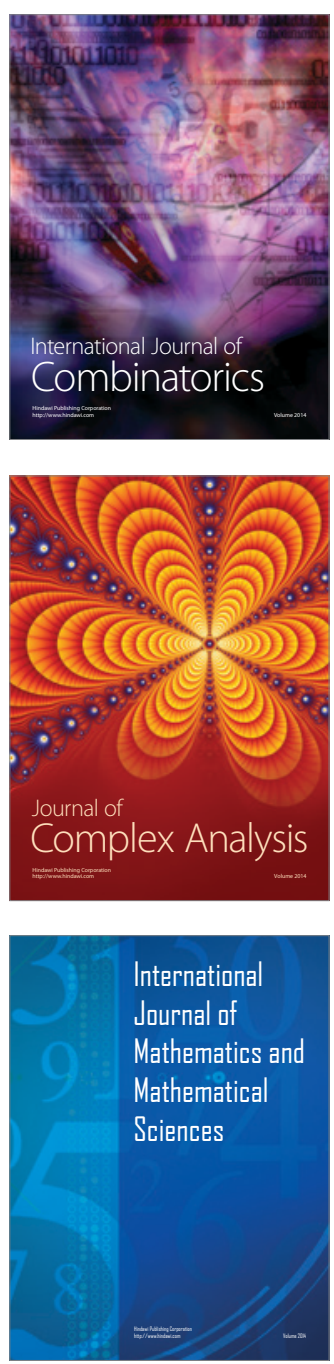
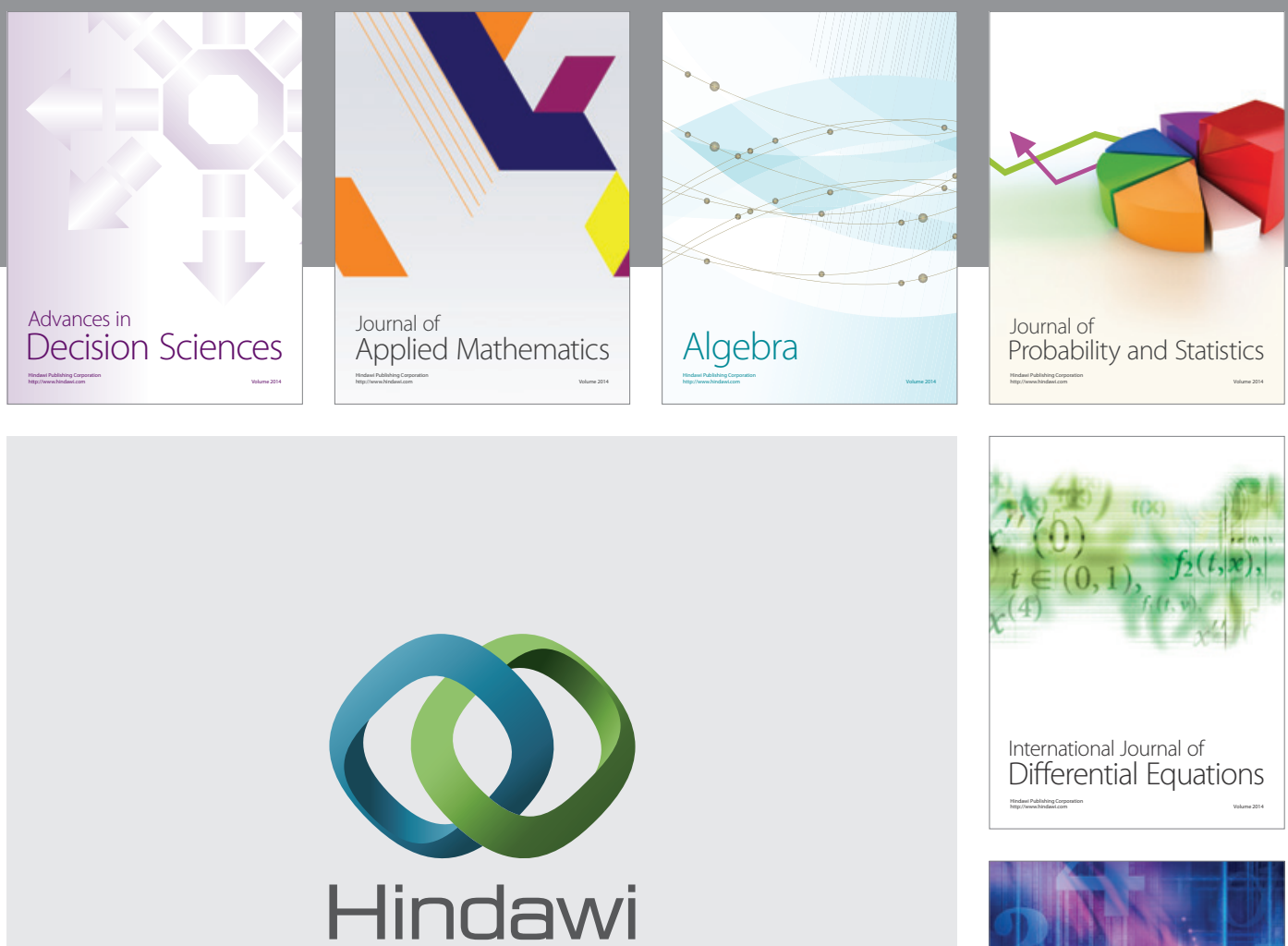

Submit your manuscripts at http://www.hindawi.com
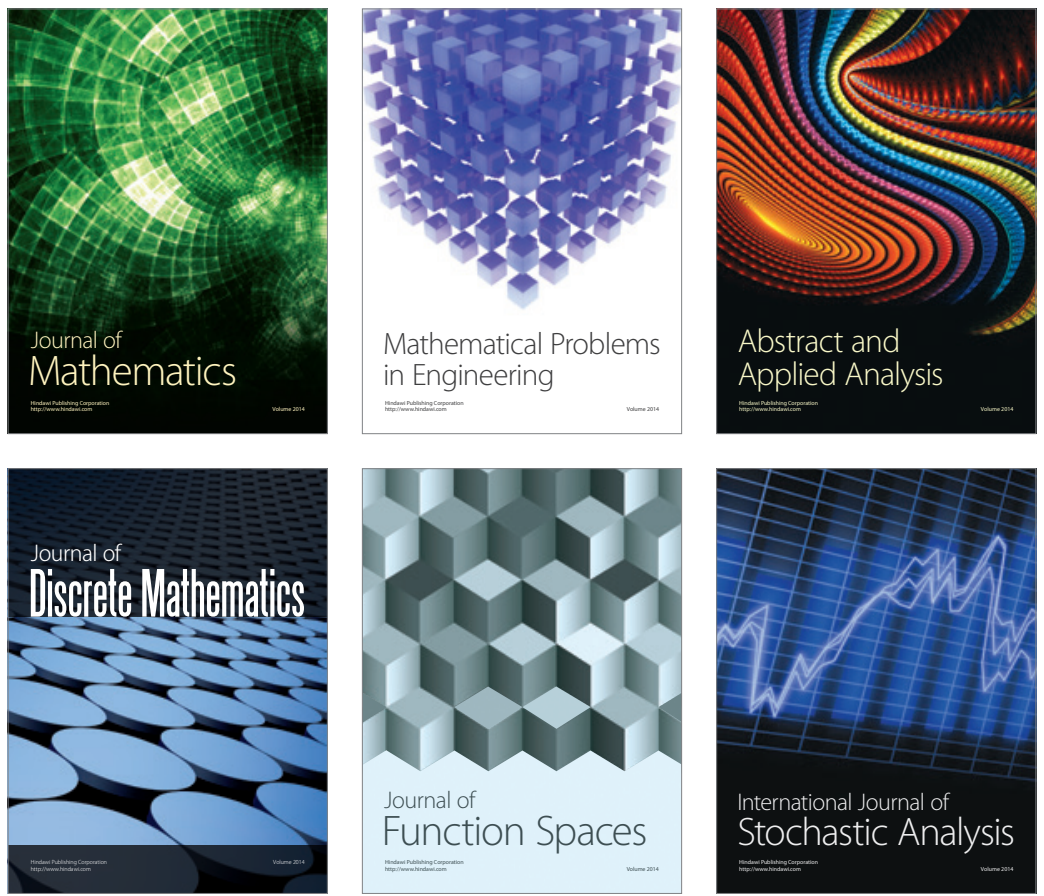

Journal of

Function Spaces

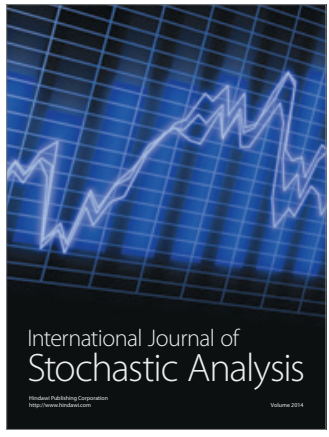

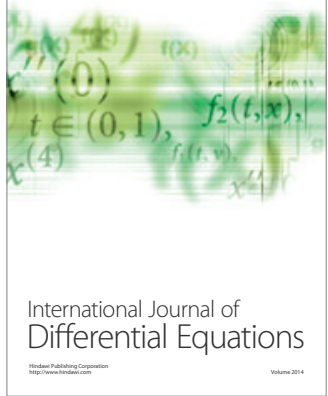
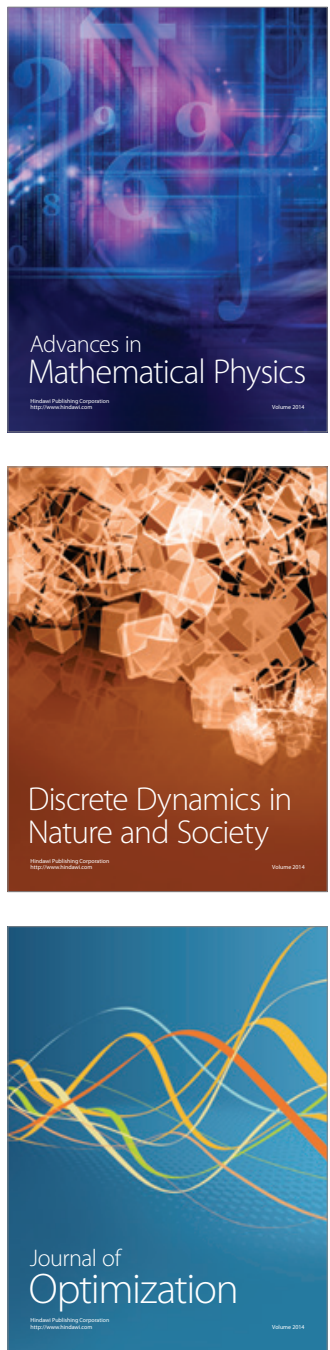